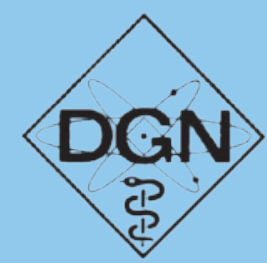

\title{
90. Geburtstag von Professor Wilhelm Finck
}

Am 3. August 2019 ist Prof. Dr. sc. med. Wilhelm Finck 90 Jahre alt geworden.

Herr Professor Finck gehört zu den bedeutenden ostdeutschen Nuklearmedizinern und war von 1969 bis 1991 Leiter der Nuklearmedizin der Universität Rostock. Zusammen mit Prof. Dr. Hans Jacob Correns (Greifswald) und Prof. Dr. Harald Frithjof Deckart (Berlin) hat er die Entwicklung der Nuklearmedizin in der DDR entscheidend beeinflusst, ebenso wie mit seinen Tätigkeiten als Vorsitzender der „Sektion Nuklearmedizin“ der Gesellschaft für medizinische Radiologie (GMR) von 1975 bis 1979 und der Gesellschaft für Nuklearmedizin (GNM) von 1983 bis 1987.

Im Jahr 1972 war Herr Professor Finck zusammen mit Höfer/Wien, Kellersohn/Paris u. a. Mitbegründer der ENMS (European Nuclear Medical Society) und von 1980 bis 1982 dann Präsident dieser Gesellschaft. Neben Prof. Dr. Friedrich Wolf (Erlangen), Prof. Dr. E. Vauramo (Finnland) u. a. war auch Professor Finck zudem entscheidend an den Vorbereitungen der späteren Fusion der beiden europäischen Gesellschaften ENMS und

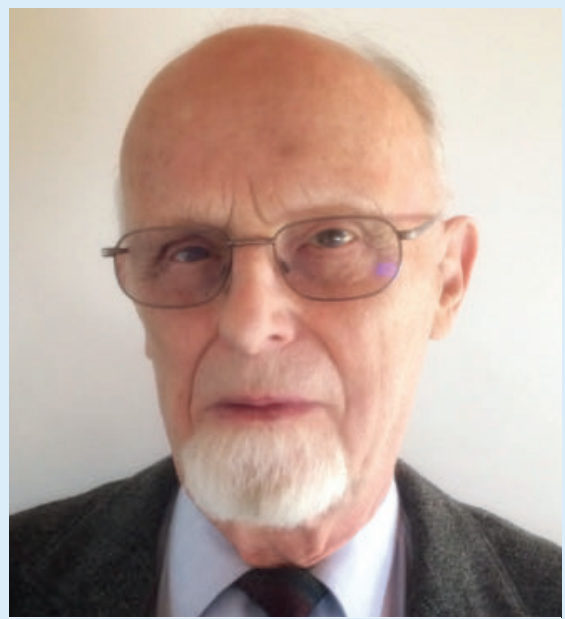

Prof. Dr. sc. med. Wilhelm Finck (Foto: privat)
SNME (Society of Nuclear Medicine Europe) zur EANM (European Association of Nuclear Medicine) beteiligt. Die Gründung der EANM erfolgte dann im Jahr 1987 in Budapest.

Den Weg der Zusammenarbeit in Wissenschaft und Ausbildung hat Herr Professor Finck auch auf nationaler und regionaler Ebene verfolgt. 1969 war er auf den ersten Lehrstuhl für Nuklearmedizin an einer (ost)deutschen Universität nach Rostock berufen worden. Gemeinsam mit Herr Professor Correns initiierte Professor Finck im Jahr 1971 zudem die Regionaltagungen Nord, in der er bis heute aktiv ist.

Herr Professor Finck hat in Halle studiert, im Jahr 1957 promoviert und 1967 in Jena seine Habilitation und Dozentur als Facharzt für Pathologische Physiologie erhalten. Seine erste Arbeit mit Radionukliden datiert von 1955. Radionuklidkinetik, Strahleneinfluss auf Stoffwechsel und Zellen, Radiomimetika, Strahlensyndrom und die Einführung nukleardiagnostischer Tests in die Klinik waren wissenschaftliche Arbeitsgebiete von ihm in Jena. Schwerpunkte in Klinik und Forschung in Rostock waren dann die Radiopharmakaentwicklung, Radionukliddiagnostik und Therapien.

Als Leiter des Forschungsverbandes „Medizinische Diagnostik und Gesundheitsschutz" des Ministeriums für Gesundheitswesen koordiniert Professor Finck von 1971 bis 1975 medizinische Forschung auf nationaler Ebene, als Direktor für Forschung des Bereichs Medizin der Uni Rostock von 1974 bis 1985 zudem auch regionale Forschungsvorhaben. Später war er von 1985 bis 1990 als Prorektor für Medizin auch für die medizinische Versorgung an der Universität verantwortlich.

Im Jahr 1991 wechselt Herr Professor Finck in den Ruhestand, den er bis heute jedoch nicht ganz erreicht hat. Das ist seinem Charakter geschuldet, seinem breiten Interessenspektrum (vom Strahlenschutz bis zur Gnotobiologie), seiner Fähigkeit und Freude am tiefgründigen analytischen Denken und koordinativem Handeln. Herr Professor Finck hat diese Fähigkeiten exzellent genutzt, als Präsident nationaler und internationaler Tagungen, in Beiräten von Zeitschriften, Organisationen und Gremien. Er hat Anerkennung und Ehrungen dafür erhalten, wie beispielsweise von der Nuklearmedizin im Jahr 1984 die Georg-von-Hevesy-Rudolf-Schönheime r-Medaille. In der Leibnitz-Sozietät (vormals Akademie der Wissenschaften der DDR) ist er noch immer engagiert.

Seine Familie fordert ihn und ist gleichsam Ruhepol und Kraftquelle in seinem Wohnsitz nahe Rostock. Sein Arbeitsplatz hat gewechselt - von der Uni-Bibliothek an den heimischen PC und ins virtuelle Web. Ehemalige Mitarbeiter, Schüler, Promovenden und die Nuklearmediziner aus Mecklenburg-Vorpommern wünschen ihrem Ehrenvorsitzenden weiterhin Gesundheit, Schaffenskraft und Lebensfreude, die bei Herr Professor Finck immer auch eine Portion intellektueller Geistesakrobatik beinhaltet.

Prof. Dr. Gerhard Kirsch (Greifswald)

Prof. Dr. Bernd Joachim Krause (Rostock)

\section{VERANTWORTLICH FÜR DEN} INHALT

\section{Deutsche Gesellschaft für} Nuklearmedizin e. V. (DGN)

Prof. Dr. Bernd Joachim Krause

(Präsident)

Nikolaistr. 29, 37073 Göttingen

E-Mail: office@nuklearmedizin.de

\section{Schriftführer:}

Prof. Dr. Peter Bartenstein

E-Mail: office@nuklearmedizin.de 